\title{
Hydrogen diffusion in high temperature proton conducting ceramics
}

\author{
S. Sorieul, S. Miro, M. Taillades-Jacquin, J. Dailly, F. Mauvy, M.-H. Berger and P. Berger
}

\section{Introduction}

High temperature proton conductors (HTPC) are envisioned as electrolytes for fuel cells working at intermediate temperature $\left(400-600{ }^{\circ} \mathrm{C}\right)$ to complement $\mathrm{Y}: \mathrm{ZrO}_{2}$ electrolytes operating at $800-$ $100{ }^{\circ} \mathrm{C}$. The most mature HTPC are doped perovskites (ABO3) where tetravalent cation $\mathrm{B}$ is partially substituted by a trivalent one [1]. Protons can be introduced in the lattice as point defects corresponding to hydroxyl groups on oxygen ion sites. In the temperature region of interest for technological applications, lattice vibrations allow the diffusion of protons by jumping and reorientation of $\mathrm{O}-\mathrm{H}$ bonds (hoping mechanism). $\mathrm{BaCeO}_{3}$ or $\mathrm{SrCeO}_{3}$-based perovskites doped with a rare earth are the most widely studied compounds [2], [3] and [4]. However the proton conductance of these ceramics and their chemical stability are lower than the calculated values on single crystals and not sufficient to fulfill technological requirements. In most cases, the reasons for these discrepancies lie in uncontrolled microstructures with inter- and intra-granular defects that act as barriers for hydrogen diffusion but are preferential paths for chemical degradation by hydrolysis or carbonation [5] and [6].

Microstructure induced limitations are usually evidenced via impedance measurements which enable determination of respective contributions of bulk and grain boundaries to overall conductivity [7], [8], [9] and [10]. Further information on hydrogen transport relevant for improvement of microstructure design requires local methods for direct hydrogen concentration measurements [11]. Nuclear microanalysis, especially ERDA technique, meets this demand. Measurement of hydrogen concentration gradients gives a true insight into transport mechanisms since various diffusion and transport models can be directly assessed. An easy method consists in achieving incomplete hydrogen loading, either by exposing a dried ceramic to a wet atmosphere or unloading a fully hydrated one under vacuum or dry atmosphere, followed by ERDA profiling of a cross section of the sample. For practical reasons, we have chosen a vacuum annealing of previously fully loaded samples.

\section{Experimental}

Disk shaped samples of composition $\mathrm{BaCe}_{0.9} \mathrm{Y}_{0.1} \mathrm{O}_{3-x}(\mathrm{BCY} 10)$ have been synthesized according two 


\section{CEA / ICGm / ICMCB @ 2008}

different process, one starting from nanometric particles (referred here as AIME) the other from a semi-commercial powder (referred as ICMCB). Both have been hydrogen loaded under wet atmosphere. As-sintered ceramics have been at first dried at $900{ }^{\circ} \mathrm{C}$ under vacuum and then exposed to $3 \% \mathrm{H} 2 \mathrm{O}$ at $600{ }^{\circ} \mathrm{C}$ for hydrogen full load. Hydrogenated samples have been then annealed for short times between 20 and $60 \mathrm{~min}$ at various temperatures under vacuum in order to induce partial hydrogen depletion. Hydrogen content has been eventually profiled by means of standard ERDA on cross sections to evidence possible hydrogen gradients induced by annealing from surface depletion.

ERDA measurements have been performed with a $3 \mathrm{MeV}^{4} \mathrm{He}$ microbeam (beam size $3 \times 3 \mu \mathrm{m}^{2}$ intensity $500 \mathrm{pA}$ ). The particle detector was placed at $30^{\circ}$ and covered with $12 \mu \mathrm{m}$ aluminium foil.

In spite of depth profiling capabilities of ERDA which enable to distinguish between surface atmospheric hydrolysis and true bulk hydration, analyzed depth is reduced to $\sim 500 \mathrm{~nm}$ under the surface. Since these perovskites are easily hydrolyzed, sample surfaces were polished with absolute ethanol and a great attention was paid to limit surface water uptake between sample preparation and putting the sample in the analysis chamber.

\section{Results}

Although estimated from bulk thermogravimetric measurements, former information looked for was a mean hydrogen content assessment. Hydrogen profiling has been at first performed on non thermally treated samples. As already mentioned, hydrogen overconcentration related to surface hydrolysis contributes to a major part of ERDA spectra but this altered layer is thinner than analyzed depth. ERDA spectrum presented on Fig. 1 shows a relatively sharp transition between surface and shallow depth hydrogen signal, the latter being considered representative of bulk content. As foreseen, line scans show slightly spread but flat profiles. Mean hydrogen contents determined from standardization with Kapton ${ }^{\circledR}$ foils are very close to thermogravimetric data which confirms that the selected region of interest in the ERDA spectra are not overlapped by surface contamination.

Temperature and duration of thermal treatments have been selected on the basis of data published by Kreuer et al. [1] with the help of a simple diffusion model based on Fick's law:

$$
\frac{C-C_{\mathrm{S}}}{C_{0}-C_{\mathrm{S}}}=\operatorname{erf}\left(\frac{x}{2 \sqrt{D t}}\right),
$$

where $\mathrm{x}, \mathrm{D}, \mathrm{t}, \mathrm{C}, \mathrm{CO}$ and CS are, respectively, depth, diffusion coefficient, time, hydrogen concentration at depth $\mathrm{x}$, starting hydrogen bulk concentration and surface concentration. For 


\section{CEA / ICGm / ICMCB @ 2008}

treatment under vacuum, the limit condition is $\mathrm{CS}=0$. This model implies that near surface hydrogen concentrations gradients should be easily visible from $200^{\circ} \mathrm{C}$ even with short thermal treatment of a few 10 min (extension of concentration gradients within 50-100 $\mu \mathrm{m}$ ). Fig. 2 and Fig. 3 summarize concentration profiles measured on cross sections of samples from two different origins, for two temperatures, 200 and $500{ }^{\circ} \mathrm{C}$.

Unexpectedly, profiles at $200^{\circ} \mathrm{C}$ are flat and the mean hydrogen level remains practically unchanged with respect to untreated samples. At $500{ }^{\circ} \mathrm{C}$, two phenomenons are observed. As foreseen, a significant decrease of hydrogen concentration is visible from the surface to the bulk with an extension of several hundreds of micrometers. Secondly, the mean level of the plateau of the core of the samples slightly decreases.

Data treatment of gradient zones by means of Eq. (1) shows a fair agreement with the Fick's law model. Fig. 4 reports both experimental data and several simulations for various values of hydrogen diffusion coefficients from $2 \times 10-7$ to $2 \times 10-5 \mathrm{~cm} 2 / \mathrm{s}$. In spite of a significant data scattering, the samples can be ranked one from the other. The mean values found, $2 \times 10-6$ and $7 \times 10-7 \mathrm{~cm} 2 / \mathrm{s}$, are relatively close to Kreuer's data, $1.95 \times 10-7 \mathrm{~cm} 2 / \mathrm{s}$ [1].

\section{Discussion}

We have learned from this very first set of experiments that long distance hydrogen transport in $\mathrm{BaCe}_{0.9} \mathrm{Y}_{0.1} \mathrm{O}_{3-x}$ can be roughly described with a Fickian model. Taken into account the usual spreading out of this kind of data, the diffusion coefficients measured for the two samples are close together. However, hydrogen transport mechanisms might be more complicated. Firstly, in the same sample, hydrogen concentration gradients are not always identical between the two sides exposed to vacuum during the hydrogen removal process. This does not seem correlated to the position of the sample in the treatment device but likely to random differences in surface properties. Surface hydrolysis is practically unavoidable which creates a transfer resistance. Depending on its magnitude, the concentration gradients should be modified in spite of identical bulk diffusion parameters. Improvement of the model by taking into account surface resistance will make sense only after acquisition of more experimental data.

The second intriguing point is the decrease of the plateau level. This behaviour cannot be reproduced from the diffusion model since a decrease of the plateau should correspond to a very high surface transfer resistance and, hence, that no concentration gradient should be observed. A tentative explanation might be the existence of two different diffusing species, more or less bonded to the oxide, the weakly bonded form being evolved very quickly at the beginning of the thermal annealing. This hypothesis will be checked later.

The most important point is that the nature of the diffusing species in unknown in this case. The 


\section{CEA / ICGm / ICMCB @ 2008}

observed hydrogen gradients might result from a dehydration process which involves hydroxyl group transport.

\section{Conclusion}

Direct investigations for hydrogen diffusion in BCY10 ceramics have shown that hydrogen gradients can be correctly described with a simple Fickian diffusion model. Diffusion coefficients determined from ERDA profiling are close to those formerly published by Kreuer. However, this model does not account for all experimental data. At least, the existence of surface transfer resistance should be taken into account but a question remains on the nature of the diffusing species. Ongoing investigations will enable to propose an enhanced model.

\section{Acknowledgement}

This work has been supported in the frame of the TECTONIC Project from PAN-H program of the National Research Agency (ANR).

\section{References}

[1] K.-D. Kreuer et al., Chem. Rev. 104 (2004), p. 4637.

[2] H. Iwahara, T. Esaka, H. Uchida and N. Maeda, Solid State Ionics 3-4 (1981), p. 359.

[3] K.D. Kreuer, Solid State Ionics 97 (1997), p. 1.

[4] T. Schober, Solid State Ionics 162-163 (2003), p. 277.

[5] M.-H. Berger, A. Sayir and P. Berger, Int. J. Hydrogen Energy 31 (2005), p. 1103.

[6] T. Norby, Br. Ceram. Proc. 63 (2003), p. 1.

[7] S.M. Haile, G. Staneff and K.H. Ryu, J. Mat. Sci. 36 (2001), p. 1149.

[8] M.-H. Berger, A. Sayir, F. Dynys and P. Berger, Solid State Ionics 177 (2006), p. 2339.

[9] U. Reichel, R.R. Arons and W. Shilling, Solid State Ionics 86-88 (1996), p. 639.

[10] De Vries, Solid State Ionics 100 (1997), p. 193.

[11] P. Berger, J.-P. Gallien and H. Khodja et al., Solid State Ionics 177 (2006), p. 1655. 
Figures

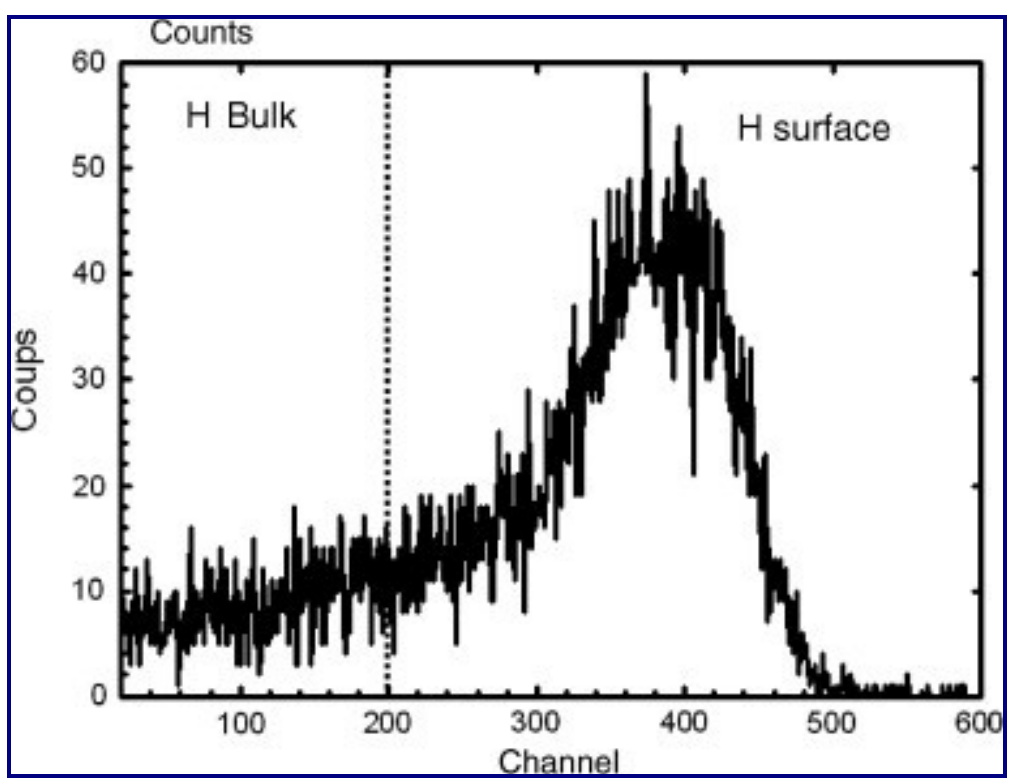

Fig. 1. Typical ERDA spectrum measured on BCY10 ceramics. The low energy region of interest is selected to calculate hydrogen concentration.

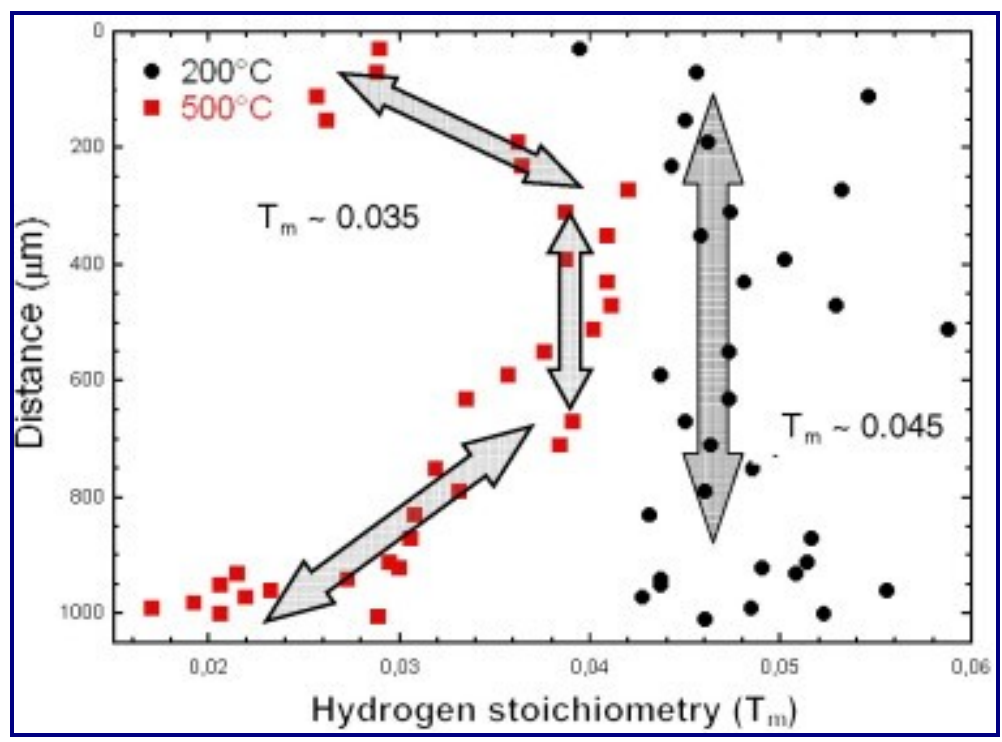

Fig. 2. Hydrogen stoichiometry profiles measured on cross sections of AIME samples for 20 min annealing at $200^{\circ} \mathrm{C}$ (circles) and $500^{\circ} \mathrm{C}$ (squares). 


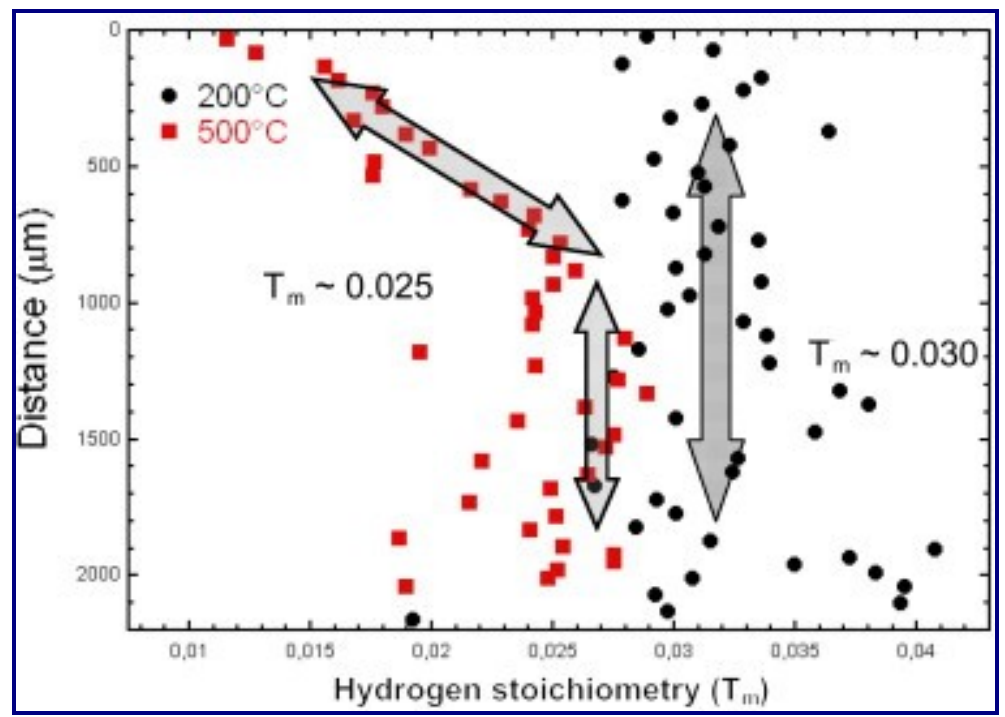

Fig. 3. Hydrogen stoichiometry profiles measured on cross sections of ICMCB samples for 20 min annealing at $200^{\circ} \mathrm{C}$ (circles) and $500^{\circ} \mathrm{C}$ (squares).

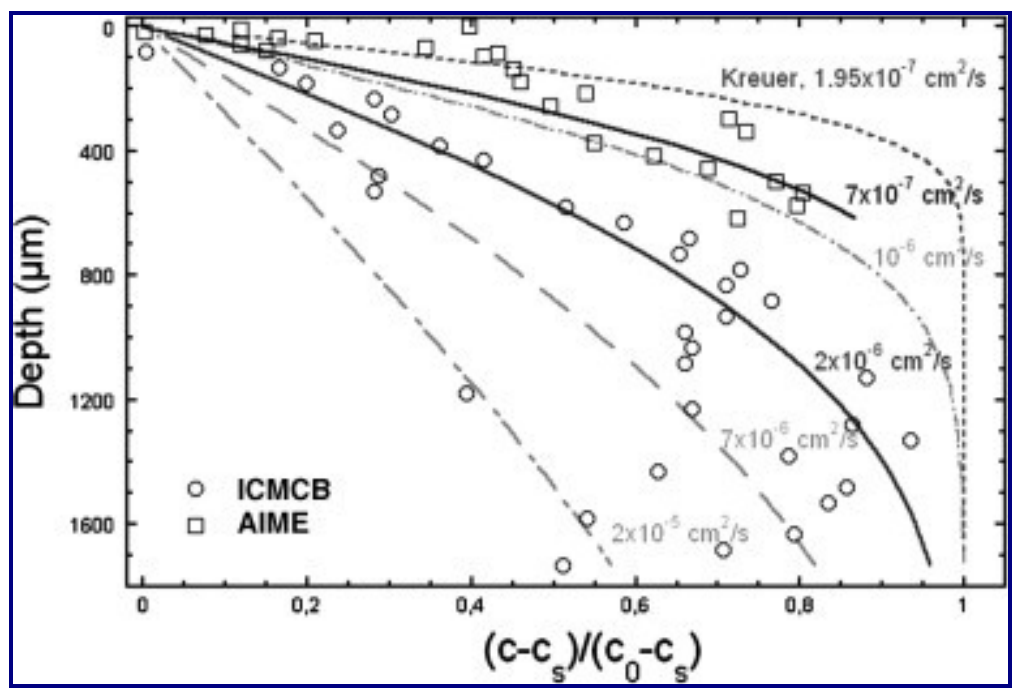

Fig. 4. Treated data according to a simple Fickian model and theoretical curves calculated for various diffusion coefficients (squares: $A$ IME, circles: ICMCB). 